\title{
Non-Basic Spin Matrices (*).
}

\author{
B. DoDDs (Edinburgh) $(*)$
}

Summary. - The spin transforming matrices of all types in three dimensions are obtained, explicitly, in each of two alternative forms, together with corresponding formulae for the inverse matrices. Similar results are obtained for the spin transforming matrices and their inverses correspond. ing to the symmetric irreducible spinors in 4-dimensional space-time.

\section{1. - Introduction.}

In $\mathrm{k}=2 v$ or $k=2 v+1$ variables, where $v$ is a positive integer, the two-valued or spin representations of the orthogonal group correspond to partitions $\left(\lambda_{1} \lambda_{2} \ldots \lambda_{\nu}\right)$ into $v$ parts, in which each part is equal to half an odd integer and $\lambda_{1} \geqslant \lambda_{2} \geqslant \ldots \geqslant \lambda_{y}$. The corresponding character is denoted by $\left[\lambda_{1} \lambda_{2} \ldots \lambda_{y}\right]$. The simplest case is that of the partition $\left(\left(\frac{1}{2}\right)^{v}\right)$, which is called the basic spin representation of the orthogonal group. A basic spinor is a vector which, corresponding to a given orthogonal transformation, is transformed by the basic spin matrix. Similarly a spinor of type $\left[\lambda_{1} \lambda_{2} \ldots \lambda_{\nu}\right]$ is a vector which, corresponding to a given orthogonal transformation, is transformed by the spin transforming matrix whose character is $\left[\lambda_{1} \lambda_{2} \ldots \lambda_{y}\right]$, i.e. the spin transforming matrix of type $\left[\lambda_{1} \lambda_{2} \ldots \lambda_{\nu}\right]$.

\section{2. - Three dimensions.}

2.1. - It is well known that in three dimensions, the $m$-th induced matrix of the basic spin matrix $U$ is equal to a matrix of type [m/2], as witnessed by the character equation

$$
[1 / 2] \otimes\{m\}=[m / 2],
$$

where $m$ is a positive integer, and the symbol $\otimes$ denotes the operation known as "plethysm", LrTrLewood [3]. One writes $U^{\{m\}}=V(m / 2)$, where $U^{\{m\}}$ is the $m$-th induced matrix of $U$ and $U(m / 2)$ represents the matrix of type [m/2]. If $m$ is taken to be an odd positive integer $(2 n+1)$ say where $n$ is a positive integer, then $U(m / 2)$ is a spin transforming matrix. One has that

$$
U^{\{2 n+1\}}=U\left(n+\frac{1}{2}\right),
$$

(*) Entrata in Redazione il 16 maggio 1972 . 
i.e. the $(2 n+1)$-th induced matrix of the basic spin matrix is equal to the spin transforming matrix of type $\left[n+\frac{1}{2}\right]$. In the ensuing sections, two alternative forms for $U\left(n+\frac{1}{2}\right)$ are obtained. Further, two forms for $U\left(n+\frac{1}{2}\right)^{-1}$, i.e. the inverse of $U\left(n+\frac{1}{2}\right)$, are also obtained.

2.2. - To fit in with the metric

$$
(d s)^{2}=\left(d x_{1}\right)^{2}+\left(d x_{2}\right)^{2}+\left(d x_{3}\right)^{2}
$$

the anticommuting set of matrices $X_{i}(i=1,2,3)$, is taken as a set of $2 \times 2$ complex matrices whose squares are $+I$. Corresponding to any orthogonal transformation,

$$
x_{i}^{\prime}=a_{i}{ }^{j} x_{j},
$$

there is a matrix $U$, the basic spin matrix, which is unique apart from sign such that

$$
X_{i}^{\prime}=a_{i}{ }^{i} X_{j}= \begin{cases}U^{-1} X_{i} U, & \text { if }\left|a_{s}{ }^{t}\right|=+1, \\ \left|a_{s}{ }^{t}\right| \cdot U^{-1} X_{i} U, & \text { if }\left|a_{s}{ }^{t}\right|=-1,\end{cases}
$$

where $A=\left[a_{s}{ }^{i}\right]$ is an orthogonal matrix.

Explicit forms for all of the irreducible spinors in three dimensions have been given elsewhere, DoDDs [2], and the same notation as used in that paper is used here. The case of an irreducible spinor of type [3/2] is considered first, to which all higher-order cases are found to be intimately related. Consider then a spinor $V_{i}$ of type [3/2] where, from [2],

$$
V_{i}=W_{i}-\frac{1}{3} X_{i}\left(X^{q} W_{q}\right)
$$

and $X^{i} V_{i}=0 . W_{i}$ is of type $\{1\}[1 / 2] . \quad V_{i}$ consists of three 2 -vectors of which, since $X^{i} V_{i}=0$, only two are independent. Take these two to be $V_{1}$ and $V_{2}$ say, then it is seen that

$$
V_{3}=X_{1} X_{3} V_{1}+X_{2} X_{3} V_{2}
$$

Hence instead of taking the spinor of type [3/2] to be $V_{i}$ with $X^{i} V_{i}=0$, it may be taken to be $V(3 / 2)$ say, where

$$
V\left(\frac{3}{2}\right)=\left[\begin{array}{l}
V_{1} \\
V_{2}
\end{array}\right]
$$

Under the orthogonal transformation, $V_{i} \rightarrow V_{i}^{\prime}$, where

$$
V_{i}^{\prime}=U a_{i}{ }^{j} V_{j}
$$


Hence after transformation, $V(3 / 2) \rightarrow V^{\prime}(3 / 2)$ where

$$
V^{\prime}\left(\frac{3}{2}\right)=\left[\begin{array}{l}
V_{1}^{\prime} \\
V_{2}^{\prime}
\end{array}\right]=\left[\begin{array}{l}
U a_{1}{ }^{j} V_{j} \\
U a_{2}{ }^{j} V_{i}
\end{array}\right]=\left[\begin{array}{c:c}
U & 0 \\
0 & C
\end{array}\right]\left[\begin{array}{c:c}
a_{11} I+a_{13} X_{1} X_{3} & a_{12} I+a_{13} X_{2} X_{3} \\
\hdashline a_{21} I+a_{23} X_{1} X_{3} & a_{22} I+a_{23} X_{2} X_{3}
\end{array}\right]\left[\begin{array}{c}
V_{1} \\
\hdashline V_{2}
\end{array}\right]
$$

since $V_{3}=X_{1} X_{3} V_{1}+X_{2} X_{3} V_{2}$ and $a_{s}^{i}=a_{s t}$. The above equation is rewritten in the form

$$
V^{\prime}(3 / 2)=U C_{1} V(3 / 2)
$$

where

and

$$
C_{1}=\left[\begin{array}{c:c}
\alpha & \beta \\
\hdashline \gamma & \delta
\end{array}\right]
$$

$$
\begin{array}{ll}
\alpha=a_{11} I+a_{13} X_{1} X_{3}, & \beta=a_{12} I+a_{13} X_{2} X_{3}, \\
\gamma=a_{21} I+a_{23} X_{1} X_{3}, & \delta=a_{22} I+a_{23} X_{2} X_{3} .
\end{array}
$$

The matrices $\alpha, \beta, \gamma$ and $\delta$ are seen to have the following properties:

$$
\alpha \gamma=\gamma \alpha, \quad \beta \delta=\delta \beta, \quad \alpha \delta+\beta \gamma=\delta \alpha+\gamma \beta .
$$

Note that neither $\alpha$ and $\beta$ nor $\gamma$ and $\delta$ commute with one another.

Hence, by definition, the spin transforming matrix of type [3/2], corresponding to the given orthogonal transformation, is equal to $U C_{1}$, i.e. $U(3 / 2)=U C_{1}$. As was seen in 2.1, this matrix must be equivalent to the third induced matrix of $U$, i.e. $U^{\{3\}}$. The matrix $U$ has been determined explicitly, in all possible cases, in terms of the $a_{s t}$ and $X_{i}$ (see, for example [1]) and hence $U(3 / 2)$ is effectively determined explicitly also.

In order to obtain $U(3 / 2)^{-1}$, the inverse of $U(3 / 2)$, it is only necessary to determine $C_{1}^{-1}$ since it is known that $U$ is unitary and hence $U^{-1}=\tilde{U}$. It is found by direct calculation that $C_{1}^{-1}=B_{1}$ where

and

$$
B_{1}=\left[\begin{array}{c:c}
\alpha_{1} & \beta_{1} \\
\hdashline \gamma_{1} & \delta_{1}
\end{array}\right]
$$

$$
\begin{array}{ll}
\alpha_{1}=a_{11} I+a_{31} X_{1}^{\prime} X_{3}^{\prime}, & \beta_{1}=a_{21} I+a_{31} X_{2}^{\prime} X_{3}^{\prime}, \\
\gamma_{1}=a_{12} I+a_{32} X_{1}^{\prime} X_{3}^{\prime}, & \delta_{1}=a_{22} I+a_{32} X_{2}^{\prime} X_{3}^{\prime} .
\end{array}
$$

It is readily seen that $\alpha_{1}, \beta_{1}, \gamma_{1}$ and $\delta_{1}$ obey the same rules as their counterparts $\alpha, \beta, \gamma$ and $\delta$ as given previously.

Hence, since $U(3 / 2)=U C_{1}$,

$$
U(3 / 2)^{-1}=C_{1}^{-1} U^{-1}=B_{1} \tilde{U} .
$$


Note here that all of this analysis is valid for orthogonal transformations of both positive and negative determinant. The only possible exception to this statement is in the actual form taken by $B_{1}$ in each of the two cases. The form given however is valid for both cases, the differences being taken care of by the $X_{i}^{\prime}$ themselves.

Spin transforming matrices of higher type are now discussed, and it is found that the matrices $C_{1}$ and $B_{1}$ just obtained have a very central role to play. Thus consider an irreducible spinor of type [5/2], $V_{i j}$ say, where, from [2],

$$
V_{i j}=W_{i j}-\frac{1}{5}\left(X_{i} X^{q} W_{q j}+X_{j} X^{q} W_{q i}\right)-\frac{1}{5} g_{i j} g^{q r} W_{q r},
$$

and $X^{i} V_{i j}=0=g^{i j} V_{i j} . W_{i j}$ is of type $\{2\}[1 / 2]$. Because of the two zero contractions, just three of the six 2-vectors which constitute $V_{i j}$ are independent. These three may be conveniently taken to be $V_{11}, V_{12}$ and $V_{22}$, whilst the other three are obtained in terms of these three by using the relations $X^{i} V_{i j}=0=g^{i j} V_{i j}$, the formulae being

$$
V_{33}=-V_{11}-V_{22}, \quad V_{31}=X_{1} X_{3} V_{11}+X_{2} X_{3} V_{12}, \quad V_{23}=X_{1} X_{3} V_{12}+X_{2} X_{3} V_{22} .
$$

The spinor of type [5/2] is therefore taken to be $V(5 / 2)$ where

$$
\widetilde{V(5 / 2)}=\left[\tilde{V}_{11}, \tilde{V}_{12}, \tilde{V}_{22}\right]
$$

Following the analysis as given in the [3/2] case, under the orthogonal transformation, $V(5 / 2) \rightarrow V^{\prime}(5 / 2)$, where

$$
V^{\prime}(5 / 2)=U C_{2} V(5 / 2)
$$

and the matrix $C_{2}$ is given by

$$
C_{2}=\left[\begin{array}{ccc}
\alpha^{2} & \alpha \beta+\beta \alpha & \beta^{2} \\
\alpha \gamma & \alpha \delta+\beta \gamma & \beta \delta \\
\gamma^{2} & \gamma \delta+\delta \gamma & \delta^{2}
\end{array}\right]
$$

It follows therefore that $U(5 / 2)=U C_{2}$. However it is seen that the second induced matrix of $C_{1}$, i.e. $O_{1}^{\{2\}}$, remembering that neither $\alpha$ and $\beta$ nor $\gamma$ and $\delta$ commute with one another, is in fact equal to $C_{2}$, the previously mentioned properties of $\alpha, \beta, \gamma$ and $\delta$ ensuring that the same induced matrix $C_{2}$, as given above, is obtained no matter in what manner, as regards ordering of factors, the construction of the induced matrix is made. Thus, $C_{2}=C_{1}^{23}$, and hence

$$
U(5 / 2)=U C_{1}^{2\}}
$$


Also,

$$
\left[C_{1}^{-1}\right]^{\{2\}}=B_{1}^{\{2\}}=\left[\begin{array}{lll}
\alpha_{1}^{2} & \alpha_{1} \beta_{1}+\beta_{1} \alpha_{1} & \beta_{1}^{2} \\
\alpha_{1} \gamma_{1} & \alpha_{1} \delta_{1}+\beta_{1} \gamma_{1} & \beta_{1} \delta_{1} \\
\gamma_{1}^{2} & \gamma_{1} \delta_{1}+\delta_{1} \gamma_{1} & \delta_{1}^{2}
\end{array}\right]
$$

However,

$$
\left[O_{1}^{-1}\right]^{\{2\}}=\left[O_{1}^{\{2\}}\right]^{-1}
$$

and hence

$$
\left[C_{1}^{2\}}\right]^{-1}=B_{1}^{\{2\}}
$$

Since

$$
U(5 / 2)^{-1}=\left[C_{1}^{2 \xi}\right]^{-1} U^{-1}
$$

it follows that

$$
U(5 / 2)^{-1}=B_{1}^{\{2\}} \tilde{\widetilde{U}}
$$

Higher order irreducible spinors, of types [7/2], [9/2], [11/2], .., can be considered with similar results, and thus the analysis immediately generalises to a spinor of type $\left[n+\frac{1}{2}\right]$. Suppose that $V_{i_{1} i_{n} \ldots i_{n}}$ is an irreducible spinor of type $\left[n+\frac{1}{2}\right]$, as given explicitly in [2], where both $X^{i_{2}} V_{i_{1} i_{2} \ldots i_{n}}$ and $g^{i_{1} i_{2}} V_{i_{1} i_{2} \ldots i_{n}}$ are zero. The spinor is taken in its alternative form, viz. $V\left(n+\frac{1}{2}\right)$, a column $2(n+1)$-vector consisting of $(n+1)$ components $V_{11 \ldots 1}, V_{11 \ldots 12}, \ldots, V_{22 \ldots 2}$, (there being $n$ suffixes in each case), where each of these components is a column 2-vector. The remaining components of $T_{i_{1} i_{n}, \ldots, i_{n}}$ are obtained in terms of these $(n+1)$ components by making use of the relations $X^{i_{1}} V_{i_{1} i_{3} \ldots i_{n}}=0=g^{i_{1} i_{2}} V_{i_{1} i_{2} \ldots i_{n}}$. The spin transforming matrix of type $\left[n+\frac{1}{2}\right]$ and its inverse, $U\left(n+\frac{1}{2}\right)$ and $U\left(n+\frac{1}{2}\right)^{-1}$ respectively, are then constructed in the manner of the preceding analysis, and one has that

$$
U\left(n+\frac{1}{2}\right)=U C_{n}=U C_{1}^{\{n\}},
$$

and

$$
U\left(n+\frac{1}{2}\right)^{-1}=\left[C_{1}^{\{n\}}\right]^{-1} U^{-1}=\left[C_{1}^{-1}\right]^{[n\}} U^{-1}=B_{1}^{\{n\}} \tilde{\bar{U}}
$$

2.3. - Alternative formulae to those just given for $U\left(n+\frac{1}{2}\right)$ and $U\left(n+\frac{1}{2}\right)^{-1}$ are now obtained. Thus suppose that $V_{i_{1} i_{2} \ldots i_{n}}$ and $Y_{i_{1} i_{2} \ldots i_{n}}$ are two irreducible spinors each of type $\left[n+\frac{1}{2}\right]$. Consider the quantity $R_{n}$ where

$$
R_{n}=\tilde{\bar{Y}}_{i_{1} i_{2} \ldots i_{n}} V_{i_{\perp} i_{\mathrm{g}} \ldots i_{n}} \text {. }
$$

Under the orthogonal transformation, $R_{n} \rightarrow R_{n}^{\prime}$ where

$$
R_{n}^{\prime}=a^{i_{1} j_{3}} \ldots a^{i_{n} j_{n}} a_{i_{1}}^{k_{1}} \ldots a_{i_{n}}{ }^{k_{n}} \tilde{\bar{Y}}_{j_{1} \ldots j_{n}} \tilde{U} U V_{k_{1} \ldots k_{n}}
$$


Since $U$ is unitary, and using the orthogonal properties of the elements of the orthogonal matrix of transformation, it follows that

$$
\begin{aligned}
& R_{n}^{\prime}=g^{j_{1} k_{1}} \ldots g^{j_{n} k_{n n}} \widetilde{\bar{Y}}_{j_{1} \ldots j_{n}} V_{k_{1} \ldots k_{n z}} \\
& =\widetilde{\bar{Y}}_{k_{1} k_{k_{2} \ldots k_{z}}} V_{k_{\mathrm{z}} k_{\mathrm{z}} \ldots k_{n}} \\
& =R_{n} \text {, }
\end{aligned}
$$

and hence the quantity $R_{n}$ is an absolute invariant. The spinors $V_{i_{1} i_{2} \ldots i_{3}}$ and $Y_{i_{1} i_{2} \ldots i_{3 x}}$ may be expressed in their alternative forms, viz. $V\left(n+\frac{1}{2}\right)$ and $Y\left(n+\frac{1}{2}\right)$ respectively, as described in 2.2. If this is done, then $R_{n}$ is expressible in the form

$$
R_{n}=\overline{\overline{Y\left(n+\frac{1}{2}\right)}} M_{n} V\left(n+\frac{1}{2}\right)
$$

where $M_{n}$ is a matrix of order $[2(n+1)]^{2}$. Hence, since it has just been proved that $R_{n}$ is an absolute invariant, it follows that

$$
\widetilde{\overline{U\left(n+\frac{1}{2}\right)}} M_{n} U\left(n+\frac{1}{2}\right)=M_{n},
$$

where $U\left(n+\frac{1}{2}\right)$ is the spin transforming matrix of type $\left[n+\frac{1}{2}\right]$ corresponding to the given orthogonal transformation. Using this equation in equations (2.2.1) and (2.2.2) gives the following alternative formulae for $U\left(n+\frac{1}{2}\right)$ and $U\left(n+\frac{1}{2}\right)^{-1}$ :

$$
U\left(n+\frac{1}{2}\right)=M_{n}^{-1} U \widetilde{\overline{B_{1}^{(n)}}} M_{n}, \quad U\left(n+\frac{1}{2}\right)^{-1}=M_{n}^{-1} \overline{\widehat{C_{1}^{(n)}}} \widetilde{\bar{U}} M_{n}
$$

The set of matrices $M_{n}$ and their inverse are easily obtained; the first two in the set are given below:

$$
\begin{gathered}
M_{1}=\left[\begin{array}{cc}
2 I & X_{1} X_{2} \\
X_{2} X_{1} & 2 I
\end{array}\right], \quad M_{1}^{-1}=\frac{1}{3}\left[\begin{array}{cc}
2 I & X_{2} X_{1} \\
X_{1} X_{2} & 2 I
\end{array}\right], \\
M_{2}=\left[\begin{array}{ccc}
4 I & 2 X_{1} X_{2} & I \\
2 X_{2} X_{1} & 6 I & 2 X_{1} X_{2} \\
I & 2 X_{2} X_{1} & 4 I
\end{array}\right], \quad M_{2}^{-1}=\frac{1}{5 \cdot 2}\left[\begin{array}{ccc}
4 I & 2 X_{2} X_{1} & -2 I \\
2 X_{1} X_{2} & 3 I & 2 X_{2} X_{1} \\
-2 I & 2 X_{1} X_{2} & 4 I
\end{array}\right] .
\end{gathered}
$$

The matrix $M_{0}$ is equal to the identity matrix, $I$.

\section{3. - Four dimensions.}

3.1. - The metric is taken to be that of space-time. Thus, to fit in with the metric

$$
\left(d s^{2}\right)=\left(d x_{1}\right)^{2}+\left(d x_{2}\right)^{2}+\left(d x_{3}\right)^{2}-\left(d x_{0}\right)^{2}
$$


the anticommuting set of matrices $X_{i}$ is taken as that used by LrTturwoon [4], viz. a set of real $4 \times 4$ matrices which satisfy

$$
\begin{array}{ll}
X_{0}^{2}=-I, & X_{i}^{2}=I, \\
\tilde{X}_{0}=-X_{0}, & \tilde{X}_{i}=X_{i},
\end{array}
$$

where $i=1,2,3$. Defining the metric tensor as

it follows that

$$
g_{i j}=g^{i j}=\left\{\begin{aligned}
0 & (i \neq j), \\
1 & (i=j ; i, j=1,2,3), \\
-1 & (i=j=0),
\end{aligned}\right.
$$

$$
X^{0}=-X_{\mathfrak{v}}, \quad X^{i}=X_{i}, \quad(i=1,2,3),
$$

and hence, in tensor notation,

$$
\left(x_{i} X^{i}\right)^{2}=\left(x^{i} X_{i}\right)^{2}=g_{i j} x^{i} x^{j}=g^{i j} x_{i} x_{j},
$$

which will be taken as the metric of space-time.

Corresponding to any Lorentz transformation, $L$ say as given by

$$
x_{i}^{t}=a_{i}{ }^{i} x_{j}, \quad\left(i=1,2,3, \frac{1}{3}\right),
$$

there is a matrix $U$, the basie spin matrix, which is unique apart from sign such that

$$
X_{i}^{i}=a_{i}{ }^{j} X_{j}=U^{-1} X_{i} U
$$

where $A=\left[a_{s}^{t}\right]$ is a Lorentz matrix, whose elements thus satisfy the "orthogonal " relations

$$
a^{k}{ }_{i} a_{k j}=g_{i j}, \quad a^{k i} a_{k}^{j}=g^{i j} .
$$

Explicit forms for all of the symmetrie irreducible spinors in four dimensions have been given elsewhere [2]. The corresponding spin transforming matrices are now obtained, the method employed being just an extension of that used in the 3-dimensional case. Moreover, the results obtained here are completely analogous to those obtained in three dimensions. It is thus found that the discussion of the case of an irreducible spinor of type [3/2,1/2] plays a central part in discussions of higher order cases. It is convenient to use much the same nomenclature as was used in the 3-dimensional ease, since it is thought that little or no confusion arises by doing this.

Consider then an irreducible spinor $V_{i}$ of type $[3 / 2,1 / 2]$ where, from [2],

$$
V_{i}=W_{i}-\frac{1}{4} X_{i} X^{q} W_{a}
$$


and $X^{i} V_{i}=0 . W_{i}$ is of type $\{1\}[1 / 2,1 / 2]$. Under $L, V_{i} \rightarrow V_{i}^{\prime}$ where

$$
Y_{i}^{\prime}=U a_{i}{ }^{j} V_{j}
$$

and $U$ is given explicitly, without exception, in [1]. The spinor $V_{i}$ consists of four 4-vectors of which just three are independent because of the zero contraction. Taking these three to be $\nabla_{1}, V_{2}$ and $V_{3}$, it follows that

$$
V_{0}=X_{1} X_{0} V_{1}+X_{2} X_{8} V_{2}+X_{3} X_{0} V_{3}
$$

The spinor of type $[3 / 2,1 / 2]$ may thus be taken in its alternative form, viz. $V(3 / 2,1 / 2)$ where

$$
\widetilde{V(3 / 2,1 / 2)}=\left[\widetilde{V}_{1}, \tilde{V}_{2}, \widetilde{V}_{s}\right]
$$

Under $L, V(3 / 2,1 / 2) \rightarrow V^{\prime}(3 / 2,1 / 2)$ where

$$
\begin{aligned}
V^{\prime}\left(\frac{3}{2}, \frac{1}{2}\right) & =\left[\begin{array}{c}
V_{1}^{\prime} \\
V_{2}^{\prime} \\
V_{3}^{\prime}
\end{array}\right]=\left[\begin{array}{l}
U a_{1}{ }^{j} V_{j} \\
U a_{2}{ }^{j} V_{j} \\
U a_{3}{ }^{j} V_{j}
\end{array}\right]=U\left[a_{s}{ }^{t} I+a_{s}{ }^{0} X_{t} X_{0}\right] V\left(\frac{3}{2}, \frac{1}{2}\right) \\
& =U\left[a_{s t} I-a_{s 0} X_{t} X_{0}\right] V\left(\frac{3}{2}, \frac{1}{2}\right) \\
& =U C_{1} V\left(\frac{3}{2}, \frac{1}{2}\right) \quad \text { say },
\end{aligned}
$$

where

$$
C_{1}=\left[C_{s t}\right]=\left[a_{s t} I-a_{s}{ }^{0} X_{t} X_{0}\right]=\left[\begin{array}{lll}
l & m & n \\
p & q & r \\
u & v & w
\end{array}\right] \quad \text { say }
$$

so that $l=C_{11}=a_{11} I-a_{10} X_{1} X_{0}$, etc. Note that $s$ and $t$ here refer to row and column respectively and $s, t=1,2,3$. It is easily seen that $l, p, u$ commute with one another, as do $m, q, v$ and also $n, r, w$. In addition,

$$
\begin{aligned}
& l q+m p=q l+p m, \quad l r+n p=r l+p n, \quad m r+n q=r m+q n, \\
& p v+q u=v p+u q, \quad p w+n=w p+u r, \quad q w+r v=w q+v r \\
& v+u m=l v+m u, \quad w l+u n=l w+n u, \quad w m+v n=m v+n v .
\end{aligned}
$$

Hence, from the above equation for $V^{\prime}(3 / 2,1 / 2)$, it follows that

$$
U(3 / 2,1 / 2)=U C_{1}
$$

where $U(3 / 2,1 / 2)$ is the spin transforming matrix of type $[3 / 2,1 / 2]$ corresponding to $L$. In order to obtain $U(3 / 2,1 / 2)^{-1}, C_{1}^{-1}$ is required, $U^{-1}$ having already been 
obtained explicitly in [1]. It is found by direct calculation that

$$
\sigma_{1}^{-1}=B_{1}=\left[a_{t s} I-a_{0 s} X_{0}^{t} X_{0}^{\prime}\right]=\left[B_{s t}\right] \quad \text { say . }
$$

Clearly the elements $B_{s t}$ obey corresponding rules to those mentioned earlier for the elements $C_{s t}$. Note that the inverse of $C_{1}$ is as given, viz. $B_{1}$, no matter what sort of Lorentz transformation is being considered. It follows that

$$
U(3 / 2,1 / 2)^{-1}=C_{1}^{-1} U^{-1}=B U^{-1} .
$$

where the exact form taken by $U^{-1}$ depends upon which of the four "pieces " of the Lorentz group the transformation $L$ belongs to [1].

Higher order cases follow as they did in the 3-dimensional case. Thus if $V_{i j}$ is an irreducible spinor of type [5/2,1/2], as given explicitly in [2], where $X^{i} V_{i j}=$ $=0=g^{i j} V_{i j}$, then one takes the spinor in its alternative form, viz. $V(5 / 2,1 / 2)$ where

$$
V\left(\widetilde{\mathbf{b} / 2, \overline{1 / 2})}=\left[\tilde{V}_{11}, \tilde{V}_{22}, \tilde{V}_{33}, \tilde{V}_{23}, \tilde{V}_{31}, \tilde{V}_{12}\right]\right.
$$

Since, under $L, V_{i j} \rightarrow V_{i j}^{\prime}$ where $V_{i j}^{\prime}=U a_{i}^{k} a_{i}^{p} V_{k p}$, it follows that $V(5 / 2,1 / 2) \rightarrow$ $\rightarrow V^{\prime}(5 / 2,1 / 2)$ where

$$
V^{\prime}(5 / 2,1 / 2)=U C_{\mathrm{a}} V(5 / 2,1 / 2)
$$

and

$$
\begin{aligned}
C_{2} & =\left[\begin{array}{llllll}
l^{3} & m^{2} & n^{2} & m n+n m & n l+l n & l m+m l \\
p^{2} & q^{2} & r^{2} & q r+r q & r p+p r & p q+q p \\
u^{2} & v^{2} & w^{2} & v w+w v & w u+u w & u v+v u \\
p u & q v & r w & q w+r v & r u+p w & p v+q u \\
u l & v m & w n & v n+w m & w l+u n & u m+v l \\
l p & m q & n r & m r+n q & n p+l r & l q+m p
\end{array}\right] \\
& =C_{1}^{\{2\}}
\end{aligned}
$$

where $C_{1}^{22}$ is the second induced matrix of the matrix $C_{1}$. Note that the previously mentioned rules obeyed by $l, m, n, p$, etc. ensure that the same induced matrix $C_{2}$, as given above, is obtained no matter in what manner, as regards ordering of factors, the construction of the induced matrix is made. It follows that

$$
U(5 / 2,1 / 2)=U C_{2}=U C_{1}^{\{2\}},
$$

where $U(5 / 2,1 / 2)$ is the spin transforming matrix of type $[5 / 2,1 / 2]$ corresponding to $L$. Further, since

$$
\left[C_{1}^{-1}\right]^{\{2\}}=\left[O_{1}^{\{2\}}\right]^{-1},
$$

it follows that

$$
\begin{aligned}
U(\tilde{5} / 2,1 / 2)^{-1} & =\left[O_{1}^{\{2\}}\right]^{-1} U^{-1} \\
& =B_{1}^{\{2)} U^{-1}
\end{aligned}
$$


since

$$
\left[C_{1}^{-1}\right]^{\{2\}}=B_{1}^{\{2\}}
$$

Similar results follow for the higher order cases, and the analysis is generalized as follows. Suppose that $V_{i_{1} i_{2} \ldots i_{n}}$ is an irreducible symmetric spinor of type $\left[n+\frac{1}{2}, \frac{1}{2}\right]$, as given explicitly in [2], where $X^{i_{1}} V_{i_{1} i_{2} \ldots i_{n}}=0=g^{i_{1} i_{2}} V_{i_{1} i_{n} \ldots i_{n}}$. The spinor $V_{i_{1} i_{2} \ldots i_{n}}$ consists of $(n+3) ! / n ! 3 ! 4$-vectors of which just $(n+2)(n+1) / 2$ are independent because of the zero contractions. Taking the spinor in the alternative form $V\left(n+\frac{1}{2}, \frac{1}{2}\right)$, where $V\left(n+\frac{1}{2}, \frac{1}{2}\right)$ is a column $2(n+2)(n+1)$-vector consisting of $(n+2)(n+1) / 2$ column 4-vectors, which may be taken as just those 4-vectors of $V_{i_{1} i_{2} \ldots i_{n}}$ in which " 0 " does not appear as one of the suffixes, the spin transforming matrix of type $\left[n+\frac{1}{2}, \frac{1}{2}\right]$ and its inverse, $U\left(n+\frac{1}{2}, \frac{1}{2}\right)$ and $U\left(n+\frac{1}{2}, \frac{1}{2}\right)^{-1}$ respectively, are then constructed in the manner of the preceding analysis and are given as follows:

$$
\begin{aligned}
U\left(n+\frac{1}{2}, \frac{1}{2}\right) & =U C_{n}=U C_{1}^{\{n\}} \\
U\left(n+\frac{1}{2}, \frac{1}{2}\right)^{-1} & =\left[C_{1}^{\{n\}}\right]^{-1} U^{-1}=\left[C_{1}^{-1}\right]^{\{n\}} U^{-1} \\
& =B_{1}^{\{n\}} U^{-1} .
\end{aligned}
$$

3.2. - Alternative formulae to (3.1.1) and (3.1.2) are now obtained. Suppose that $V_{i_{1} i_{2}, i_{n}}$ and $Y_{i_{1} i_{2} \ldots i_{n}}$ are two irreducible spinors, each of type $\left[n+\frac{1}{2}, \frac{1}{2}\right]$. Consider the quantity $R_{n}$, which is bilinear in the spinors and of rank zero, where

$$
R_{n}=\tilde{Y}^{i_{1} i_{2} \ldots i_{n}} T \gamma^{r}{ }_{i_{1} i_{z} \ldots i_{n}}
$$

and $T$ is the matrix $X_{0}$ of the set of anticommuting matrices $X_{i}$. Under $L, R_{n} \rightarrow R_{n}^{\prime}$ where

$$
R_{n}^{j}=a^{i_{1} j_{1}} \ldots a^{i_{n} j_{n}} a_{i_{1}}{ }^{k_{1}} \ldots a_{i_{n}}{ }^{k_{n}} \tilde{Y}_{j_{1} \ldots i_{n}} \tilde{U} T U V_{k_{2} \ldots k_{n}}
$$

Using the "orthogonal" properties of the elements of the Lorentz matrix, it follows that

$$
\begin{aligned}
R_{n}^{\prime} & =g^{j_{1} k_{1}} \ldots g^{i_{n} k_{n}} \widetilde{Y}_{j_{1} \ldots j_{n}} \tilde{U} T U V_{k_{1} k_{2} \ldots k_{n}}, \\
& =\tilde{Y}^{k_{1} k_{2} \ldots k_{n}} \tilde{U} T U V_{k_{2} k_{2} \ldots k_{n}} .
\end{aligned}
$$

But it has been proved in [1], that

$$
\tilde{U} T C=T^{r} \quad \text { if } L=L(\div,+) \text { or } L(-,+),
$$

and

$$
\tilde{U} T U=-T \quad \text { if } L=L(+, \rightarrow \text { or } L(-,-),
$$


where $L(+,+), L(+,-), L(-,+)$ and $L(-,-)$ denote transformations belonging to the four separate "pieces * of the Lorentz group, the first sign denoting whether the determinant of the transforming matrix is positive or negative, whilst the second sign denotes whether the transformation leaves unchanged, + , or reverses, - , the direction of the time-axis. It follows therefore that

$$
R_{n}^{\prime}=\left\{\begin{aligned}
R_{n} & \text { if } L=L(+,+) \text { or } \quad L(-,+) \\
-R_{n} & \text { if } L=L(+,-) \text { or } \quad L(-,-)
\end{aligned}\right.
$$

Thus $R_{n}$ is an absolute invariant if $L=L(+,+)$ or $L(-,+)$, and is a relative invariant if $L=L(+, \rightarrow)$ or $L(-,-)$.

The spinors can be taken in their alternative forms, viz. $V\left(n+\frac{1}{2}, \frac{1}{2}\right)$ and $I^{r}\left(n+\frac{1}{2}, \frac{1}{2}\right)$, as described in 3.1. If this is done, then $R_{n}$ may be expressed in the form

$$
R_{n}=Y \widetilde{\left(n+\frac{1}{2}, \frac{1}{2}\right)} T M_{n} V\left(n+\frac{1}{2}, \frac{1}{2}\right)
$$

where $M_{n}$ is a matrix of order $[2(n+2)(n+1)]^{2}$. Hence in the light of the invariant qualities of $R_{n}$ just proved, it follows that

$$
U\left(\widetilde{\left.n+\frac{1}{2}, \frac{1}{2}\right)} T M_{n} U\left(n+\frac{1}{2}, \frac{1}{2}\right)=\left\{\begin{aligned}
T M_{n} & \text { if } L=L(+,+) \text { or } L(-,+) \\
-T M_{n} & \text { if } L=L(+,-) \text { or } L(-,-)
\end{aligned}\right.\right.
$$

and hence, using (3.1.1) and (3.1.2), one has that

$$
\begin{aligned}
& U\left(n+\frac{1}{2}, \frac{1}{2}\right)=\mp M_{n}^{-1} T \widetilde{U}^{-1} \widetilde{B_{1}^{\{n\}}} T M_{n}, \\
& C\left(n+\frac{1}{2}, \frac{1}{2}\right)^{-1}=\mp M_{n}^{-1} T \widetilde{C_{1}^{\{n\}}} \tilde{U} T M_{n},
\end{aligned}
$$

the upper signs being taken if $L=L(+,+)$ or $L(-,+)$, the lower ones if $L=$ $=L(+,-)$ or $L(-,-)$. In fact the ambiguity of sign in the above formulae is superfluous in the light of the known ambiguity of sign in spin representations. The set of matrices $M_{n}$ and their inverses ar easily obtained; e.g. $M_{0}$ is equal to the identity matrix, whilst $M_{1}$ and its inverse are as follows:

$$
M_{1}=\left[\begin{array}{ccc}
2 I & X_{1} X_{2} & X_{1} X_{3} \\
X_{2} X_{1} & 2 I & X_{2} X_{3} \\
X_{3} X_{1} & X_{3} X_{2} & 2 I
\end{array}\right], M_{1}^{-1}=\frac{1}{4}\left[\begin{array}{ccc}
3 I & X_{2} X_{1} & X_{3} X_{1} \\
X_{1} X_{2} & 3 I & X_{3} X_{2} \\
X_{1} X_{3} & X_{2} X_{3} & 3 I
\end{array}\right]
$$

4. - The author wishes to thank Professor D. E. LITTLEw ood for his continued interest during the course of this work. Thanks are also due to the Science Research Council for their award of a Research studentship during part of the course of this work. 


\section{REFERENCES}

[1] B. Donos, Basic spin matrices, Ann. di Mat., 88 (1971), pp. 333-348.

[2] B. Dodds, Symmetric spinors in k-dimensions, Ann. di Mat. 92 (1972), p. 337.

[3] D. E. Litruewood, Invariant-theory, tensors and group characters, Phil. Trans. Roy. Soc. (A), 239 (1944), p. 305.

[4] D. E. LITTLwoon, Relativity and quantum theory. A unitied model, Ann. di Mat,, 81 (1969), pp. 111. 